\title{
The value of myocardial MIBI washout rate in risk stratification of coronary artery disease
}

\author{
Mohammed Omar Mohammed Othman ${ }^{1 *}$ (D), Hosna Mohammed Moustafa', \\ Mohammed Mahmoud Abd El-Ghany² and Shaimaa Ahmed Abd El-Mon'em El-Rasad
}

\begin{abstract}
Background: Although it is well established that MIBI does not redistribute as thallium within the myocardium, it showed a reverse redistribution phenomenon that can be expressed by the rate of myocardial MIBI washout. The aim in this study was calculating the global myocardial washout of the MIBI (GWR) in patients diagnosed with coronary artery disease (CAD) of different risk stratifications.
\end{abstract}

Results: This prospective study included 100 patients. All patients were stratified into low-, intermediate-, and highrisk groups according to clinical evaluation using Framingham score, stress ECG results using Duke's score and finally myocardial perfusion imaging prognostic findings. GWR was estimated in each of these groups. GWR mean was $9.5 \%, 13 \%$, and $18 \%$ within clinically stratified patients into low-, intermediate-, and high-risk patients respectively with correlation coefficient 0.4 . In addition, GWR mean was $9.7 \%, 15.4 \%$, and $18.7 \%$ within patients stratified according to exercise ECG findings into low-, intermediate-, and high-risk patients respectively with correlation coefficient 0.6. Combining all myocardial perfusion findings, GWR mean was 7.9\%, 15.1\%, and 19.3\% in patient with low-, intermediate-, and high-risk imaging findings respectively with correlation coefficient 0.71 .

Conclusion: GWR is positively correlated with the risk stratifications of the CAD patients. GWR can be used as an additional parameter to assess the risk of CAD patients.

Keywords: Risk stratification, GWR, Framingham, Duke's score

\section{Background}

CAD is a chronic condition affecting large numbers of people across the world. Hence, the assessment of the risk and actual burden of disease is the determinant key for the management with other interventions improving the quality of a patient's life [1].

It is well noted now that the mortality rate owing to CAD has decreased, and this is a result of better detection of CAD patients, assessment of their risk, and better subsequent treatment [2].

\footnotetext{
*Correspondence: m.omarosman@yahoo.com; m.omarosman@cu.edu.eg ${ }^{1}$ Nuclear Medicine, El-Kasr Al-Ainy Cairo University, 20 El-Enshirah El-Kabeer street, El-Mohandeseen, Giza, Egypt

Full list of author information is available at the end of the article
}

Single-photon computed tomography (SPECT) myocardial perfusion scan using $\mathrm{Tc}^{99 \mathrm{~m}}$ MIBI is considered one of the main non-invasive diagnostic tools that provide valuable information in assessment of CAD [3].

It is well known that MIBI, being lipophilic, enters the myocardial mitochondria passively then, being a cation, it is trapped inside by membrane negative potential. With impaired respiratory chain resulted from repeated ischemic changes, the mitochondrial membrane partially losses its negative charges with subsequent enhanced MIBI washout [3].

Aim of the work is calculation of GWR in CAD patients with different risk stratifications.

\section{Springer Open}

(c) The Author(s). 2021 Open Access This article is licensed under a Creative Commons Attribution 4.0 International License, which permits use, sharing, adaptation, distribution and reproduction in any medium or format, as long as you give appropriate credit to the original author(s) and the source, provide a link to the Creative Commons licence, and indicate if changes were made. The images or other third party material in this article are included in the article's Creative Commons licence, unless indicated otherwise in a credit line to the material. If material is not included in the article's Creative Commons licence and your intended use is not permitted by statutory regulation or exceeds the permitted use, you will need to obtain permission directly from the copyright holder. To view a copy of this licence, visit http://creativecommons.org/licenses/by/4.0/. 


\section{Methods}

\section{Study design and population}

This prospective control study was performed in Nuclear Medicine Department in Kasr Alainy Hospital from March 2017 until December 2018. It included 100 patients [76 males and 24 females] with $54.2 \pm 8.9$ years mean age. Tc ${ }^{99 m}$ MIBI myocardial perfusion gated SPEC $\mathrm{T}$ imaging was done for all patients.

\section{Inclusion criteria}

CAD patients with different risk stratifications referred for myocardial perfusion scan.

\section{Exclusion criteria}

We excluded patients who underwent pharmacological stress study, as Duke's criteria cannot be applied.

\section{Clinical risk stratifications}

All patients were stratified clinically through the total points received according to the primary version of Framingham risk score into low-, intermediate-, and high-risk patients. For female patients, total points of 19 or less was considered low risk, from 20 to 23 points was considered intermediate risk and above 23 points was considered high risk. While for male patients, total points of 12 or less was considered low risk, from 12 to 15 points was considered intermediate risk and above 15 points was considered high risk [4].

\section{Exercise stress study results}

All patients were then separately stratified by the exercise stress results through the total points received according to Duke's risk score into low-, intermediate-, and high-risk patients. The Duke Treadmill Score (DTS) was calculated as follows:

$$
\begin{aligned}
\text { DTS }= & \text { Exercise time (in minutes }) \\
& -(5 \times \text { ST deviation in } \mathrm{mm}) \\
& -(4 \times \text { angina index })
\end{aligned}
$$

The exercise time was based on using the Bruce protocol. ST deviation referred to maximum ST change (elevation or depression) in any lead except lead aVR. The angina index was 0 points if no chest pain occurs, 1 point if non-limiting chest pain occurs, and 2 points if typical anginal pain occurs that limits exercise. Patients were categorized as low risk (score $>5$ ), intermediate risk (score between 4 and - 11), and high risk (score < - 11) [5].

\section{Myocardial perfusions scan (MPS)}

Two days protocol was used, rest in 1 day and stress exercise in the other. Additional delayed image was done following the rest study.

\section{Imaging protocol used}

Patients were instructed to fast for 4 to $6 \mathrm{~h}$ before the test. Cardiac medication including beta blockers, theophylline derivatives, nitrates, and calcium channel blockers were stopped $48 \mathrm{~h}$ prior to the study. Fatty foods (egg, milk, or chocolate) were given 15 min after $\mathrm{Tc}^{99 \mathrm{~m}}$ MIBI injection to facilitate liver and biliary system clearance. A dose of $15 \pm 3 \mathrm{mCi}(555 \pm 111 \mathrm{MBq})$ of $\mathrm{Tc}^{99 \mathrm{~m}}$ MIBI was injected intravenously for each study.

Rest study included two images; one at 60-90 min post-injection and the other delayed image was $4 \mathrm{~h}$ after the same injection. The stress study was done as usual at 30-45 min after injection.

Dual head SPECT gamma camera was used with the two heads perpendicular to each other. High-resolution low energy collimator was used. Photon peak was adjusted at $140 \mathrm{keV}$ with window $20 \%$ and 1.3 zoom factor and matrix $64 \times 64$. Patient was supine with elevated left arm to decreased attenuation. Imaging from $45^{\circ}$ right anterior oblique to $135^{\circ}$ left posterior oblique position. Synchronized ECG data with $\mathrm{R}$ wave trigger, 8 frames per cardiac cycle to produce 32 projections, each was $40 \mathrm{~s}$.

Reconstruction was done with filtered back projection and Butterworth filter. All raw data sets were corrected with isotope decay factor and checked for patient motion by reviewing a rotating cine display.

\section{Imaging interpretation}

Perfusion defects in stress images were interpreted qualitatively in terms of their location, size, and severity. Location was interpreted according to coronary vascular territories likely to be involved. Size was interpreted according to the number of segments within affected wall. Small was when the number of affected segments is less than one thirds of the total wall segments, moderate was when the number of affected segments is more than one third but less than two thirds of the total wall segments while severe was when the number of affected segments is more than two thirds of the total wall segments). Severity was interpreted according to colon display (mild, moderate, and severe).

The reversibility of the stress-induced defects was assessed in the rest images in terms of complete (became normally perfused), partial (showed perfusion improvement but not return normal), or absent (fixed perfusion defects in both stress and rest studies).

Semi-quantitative data was done using two-dimensional polar map display showing apex at center and the base of the ventricle at the periphery. Then, it was divided into 17 segments representing the whole the myocardium. apex in the center, the four apical segments as a first ring, the six 
mid-cavity segments as the second ring, and the six apical segments as the outermost ring.

Each segment was scored visually based on the tracer uptake as 0 , normal; 1 , mildly reduced; 2 , moderately reduced; 3 , severely reduced; and 4 , absent tracer uptake in rest and stress images. A summed rest score (SRS) and summed stress score (SSS) were obtained by adding the scores of the 17 segments of the rest and stress images, respectively. The summed difference score (SDS) was determined by subtracting the SRS from the SSS [6].

Gated data used for quantitative results including end systolic volume, end diastolic volume, and ejection fraction of left ventricle. Stress-induced transient ischemic dilatation was also identified [6].

\section{MPS risk stratification}

All patients were classified into three groups according to MPS findings as follows [7]:

High risk group patients have one or more of these findings: stress-induced large perfusion defect $(>20 \%$ of total left ventricular volume), stress-induced multiple perfusion defects of moderate size, severe resting left ventricular dysfunction (LV EF $<35 \%$ ), and SSS > 13 . Intermediate risk group, patients have one or more of these findings: stress-induced moderate (10-20\%) perfusion defect, mild/moderate resting LV dysfunction (LV $\mathrm{EF}=35-49 \%)$, and SSS 8-13. Low risk group, patients have all these findings: normal or small myocardial perfusion defect ( $<10 \%$ of total left ventricular volume), normal left ventricular function (LV EF $50 \%$ or more), and SSS $<8$.

\section{Calculation of GWR}

Calculation of GWR was calculated for each patient using counts per pixel in the two-dimensional polar map display as follows.

$$
G W R=\frac{\mathrm{Ce}-(\mathrm{Cd} \times \text { decay factor })}{\mathrm{Ce}} \times 100 \%
$$

$$
\begin{aligned}
& \text { Ce: total myocardial counts in early imaging } \\
& \text { Cd: myocardial counts in delayed imaging } \\
& \text { Decay factor: }=1 /(1 / 2)^{x}, x=(\text { time difference }) / 6
\end{aligned}
$$

\section{Statistical methods}

Data were statistically described in terms of mean \pm standard deviation $( \pm \mathrm{SD})$, median and range, or frequencies (number of cases) and percentages when appropriate. Comparison of numerical variables between the study groups was done using Kruskal-Wallis test with post hoc multiple 2-group comparisons. Correlation between various variables was done using Pearson moment correlation equation for linear relation of normally distributed variables and Spearman rank correlation equation for non-normal variables/non-linear monotonic relation. Stepwise multivariate regression analysis was performed to examine the potential interactions among the entered covariates. The Student $t$ test was used for comparison of paired data, and $P$ values less than 0.05 was considered statistically significant. All statistical calculations were done using computer program IBM SPSS (Statistical Package for the Social Science; IBM Corp, Armonk, NY, USA) for Microsoft Windows.

\section{Results}

\section{Characteristics of the patients}

The study included 100 patients, [76 males and 24 females with mean age $54.25 \pm 8.9$ years, maximum age was 78 years and minimum age 35 years. Fifty-four patients were hypertensive, 41 patients were diabetic, 36 patients were smoker, and 39 patients were dyslipidemic.

\section{Descriptive data}

Within the 100 patients according to Framingham score, 15,55 , and 30 patients were considered as low-, intermediate-, and high-risk respectively. Within the 100 patients according to Duke's risk score, 37, 43, and 20 patients were considered as low-, intermediate-, and highrisk respectively. Scintigraphic myocardial perfusion findings within the 100 patients demonstrated 43 patients had perfusion abnormality in less than two segments (small perfusion defects), 29 patients in 2-8 segments (moderate perfusion defects), and 28 patients in more than 8 segments (large perfusion defects). Forty-three patients had SSS less than 8, 15 patients had SSS between 8 and 13, and 42 patients had SSS more than 13. Twelve patients with global myocardial had impaired motions. Sixty-three patients had EF more than or equal 50,12 patients had EF between 49 and 35, while 25 patients had EF less than 35 . Combining all myocardial perfusion findings of the 100 patients, 40, 30, and 30 patients were considered as low-, intermediate-, and high-risk respectively.

According to Framingham scoring system, the mean GWR was $9.5 \%$ within 15 low-risk patients, $13 \%$ within 55 intermediate-risk patients, and $18 \%$ within 30 highrisk patients. According to Duke's risk score, the mean GWR was $9.7 \%$ within 37 low-risk patients, 15.4 within 43 intermediate-risk patients, and 18.7 within 20 highrisk patients. Combining all myocardial perfusion findings, the mean GWR was $7.9 \%$ within 46 patients with low-risk scintigraphic features, $15.1 \%$ within 30 patients with intermediate-risk scintigraphic features, and 19.3\% within 24 patients with high-risk scintigraphic features.

\section{Analytical data}

GWR was found to be intermediately positively correlated with the clinical risk and exercise ECG risk 
stratifications with correlation coefficient 0.4 and 0.6 respectively and strongly positively correlated with myocardial perfusion imaging poor prognostic features with correlation coefficient 0.71 (Figs. 1, 2, and 3).

GWR was negatively correlated with the ejection fraction with correlation coefficient - 0.4 (Fig. 4) and strongly positively correlated with the number of affected segment and summed difference score with correlation coefficient 0.7 and 0.76 respectively (Figs. 5 and 6).

Case presentation is found in Figs. 7 and 8.

\section{Discussion}

CAD is a chronic condition affecting large numbers of people across the world accurate evaluation of CAD would greatly increase the survival rate of CAD patients [1].

The ultimate goal is identifying those patients at high risk of adverse outcomes who may benefit from revascularization procedures as well as identification of a lowrisk population to avoid unnecessary invasive tests and procedure [8].

The ESC guidelines on the management of stable CAD define high event risk patients as those with annual mortality $>3 \%$ and low-risk patients with annual mortality $<1 \%[9]$.

We aimed in the present study to estimate MIBI GWR in CAD patients with different risk stratifications according to Framingham score, Duke's score, and myocardial perfusion findings so that GWR can be an additional prognostic parameter that aid in decision making in patient with CAD.

Richter et al. was first to explain the accelerated MIBI washout in 1995 on 36 patients with CAD. They calculated the GWR after 120 min. In 35 of 114 segments, the score improved within $120 \mathrm{~min}$ so that $69.9 \%$ $\pm 22.5 \%$ to $74.5 \% \pm 20.8 \%(P<0.01)$. In 7 of 114 segments, the score deteriorated within $120 \mathrm{~min}$ so that $85.6 \% \pm 9.9 \%$ to $80.1 \% \pm 10.7 \%(P<0.02)$. They concluded that some early defects might show changes in the late phase, such that some defects improved and other defects became worse. The major drawback in the Richter's study was using the planar image for quantitative assessment. This caused distortion and decreased accuracy of his results [10].

Khandaker et al. attempted to resolve the uncertain rating assigned to SPECT MPI in risk assessment of CAD in asymptomatic patients. Two hundred and sixty asymptomatic patients with mean age $67 \pm 8$ years and without known CAD were included in the study. Survival at 10 years was $79 \%$ in patients with normal or low-risk cardiac scan. They concluded that SPECT MPI could accurately detect and stratify asymptomatic patients. They concluded that mortality was $4.0 \%$ per year in patients with high-risk scans compared to $1.6 \%$ patients with normal scans [11].

In the present study, the analysis of myocardial perfusion SPECT in the study group included qualitative, semi-quantitative, and quantitative data. Qualitative findings showed 43 patients had perfusion abnormality in less than two segments (small perfusion defects), 29 patients in 2-8 segments (moderate perfusion defects), and 28 patients in more than 8 segments (large perfusion defects). Fifty patients showed normal wall motion, 38 patients with regional wall abnormality, and 12 patients with global myocardial impaired motions.

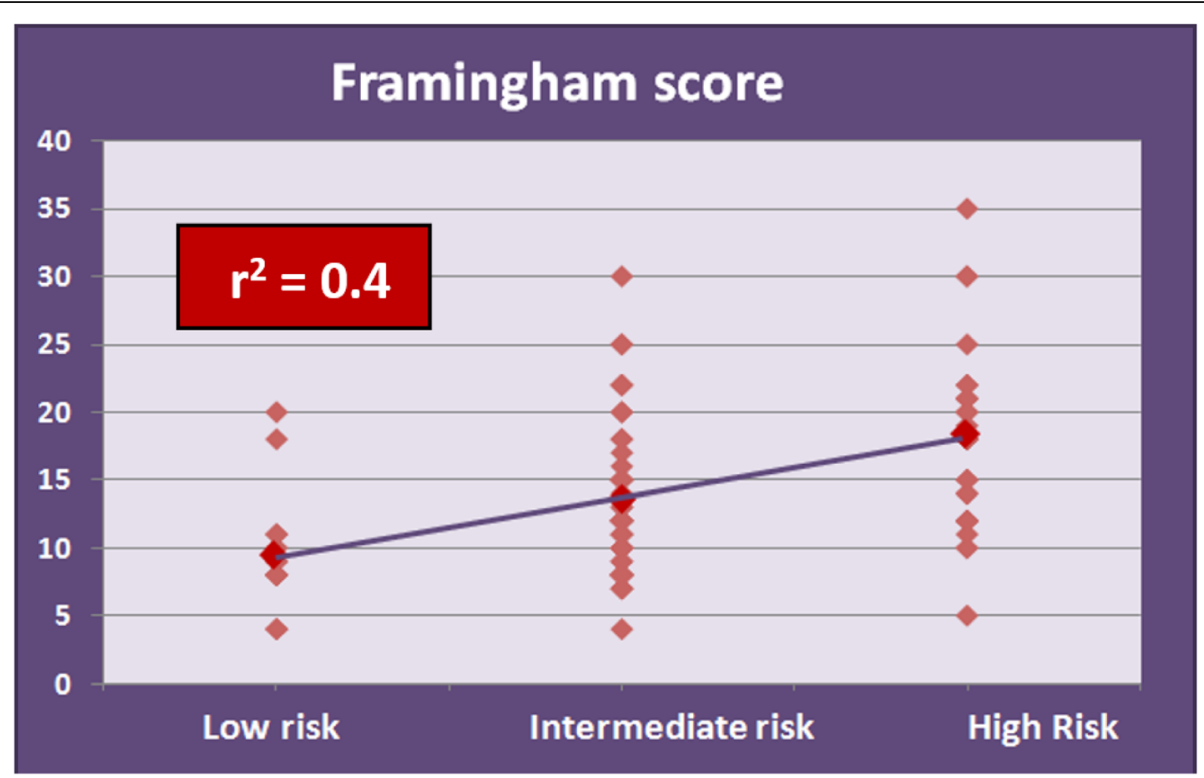

Fig. 1 GWR correlation with Framingham's score 


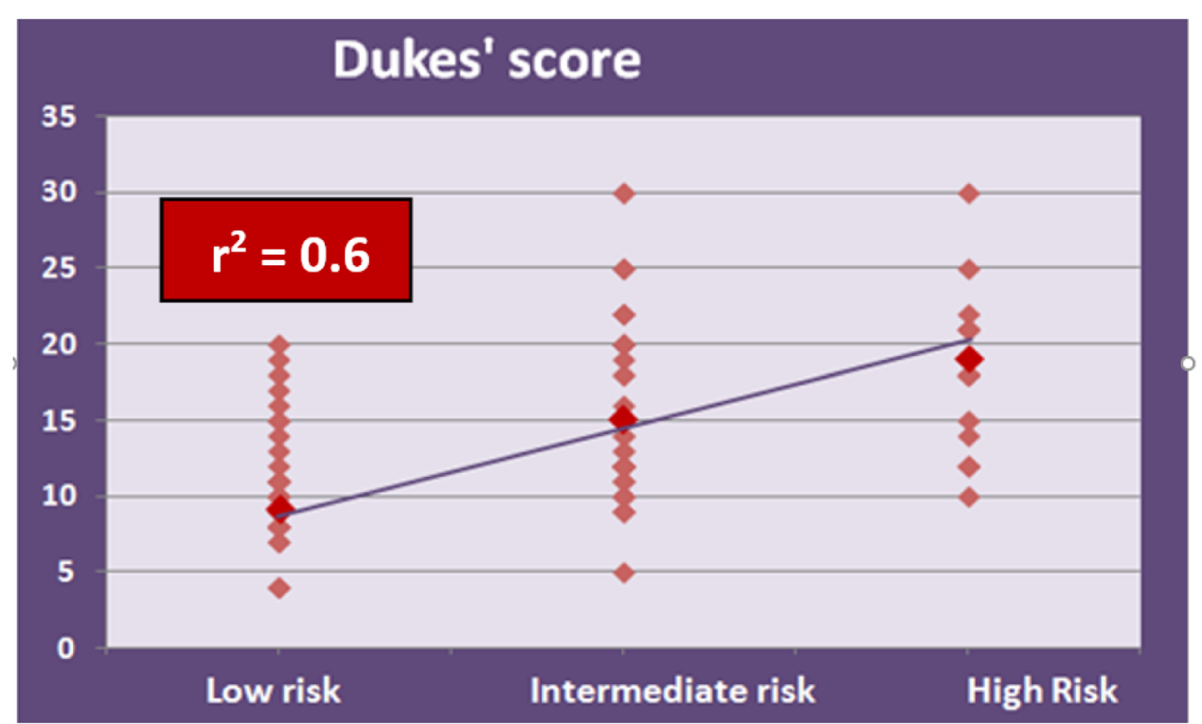

Fig. 2 GWR correlation with Duke's score

America et al. studied the additive prognostic value of perfusion and functional data of quantitative gated SPECT in 453 consecutive patients followed for average 1.33 years. Their result included 236 patients had an abnormal study, of whom 27 patients experienced serious cardiac events and 47 patients experience non-serious cardiac events and the rest witnessed no specific cardiac event. They concluded that perfusion and functional parameters derived from quantitative gated SPECT imaging could adequately be used for cardiac risk assessment. EF 52\% or SSS > 22 are at increased risk for subsequent hard events. Patients with an SSS > 14 are at increased risk for at least non-serious cardiac events [12].
In the present study, semi-quantitative and quantitative scintigraphic findings showed 38 patients with SDS less than 2, 28 patients with SDS from 2 to 4 , and 34 patients with SDS more than 4 and 34 patients with SDS more than 4. Also, 63 patients had normal EF being more than or equal $50 \%$, while 12 patients had EF from 35 to 49 . Only 25 patients had low EF less than $35 \%$. Combining all myocardial perfusion findings and semi-quantitative parameters showed that 46 patients considered as low risk, 30 patients were considered intermediate risk, and 24 patients were considered high risk. The GWR was positively correlated with correlation coefficient of 0.71 .

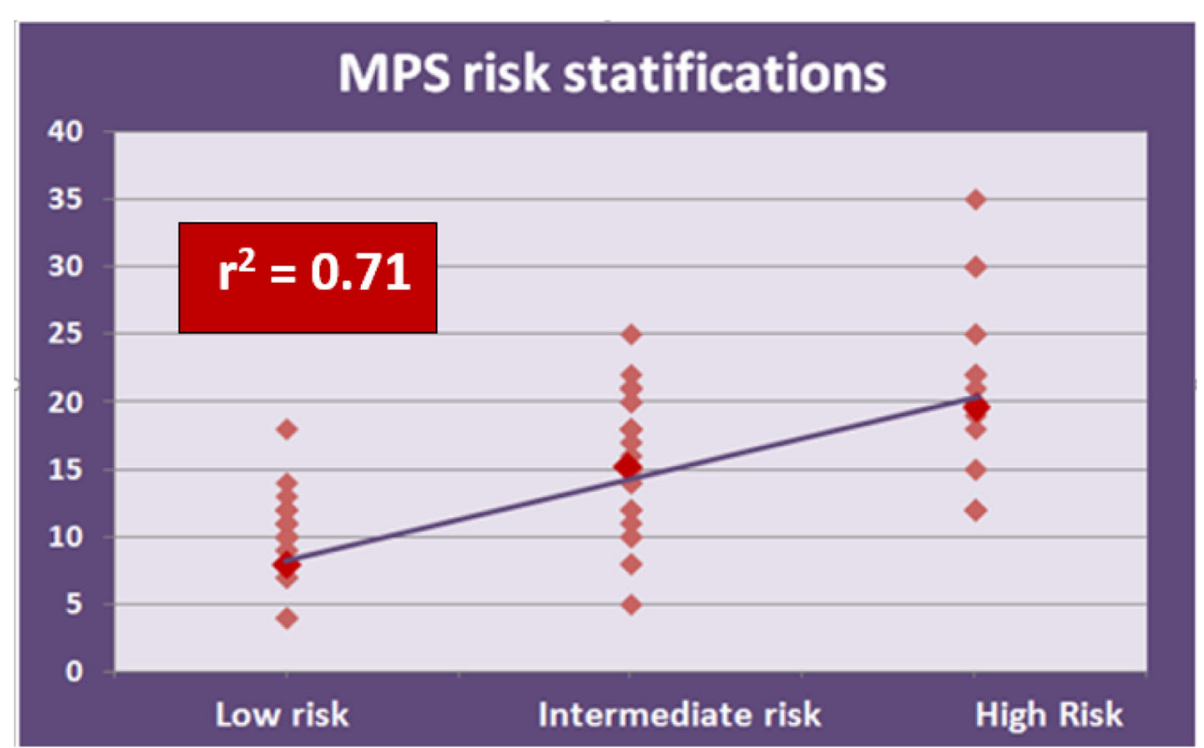

Fig. 3 GWR correlation with MPS stratification 


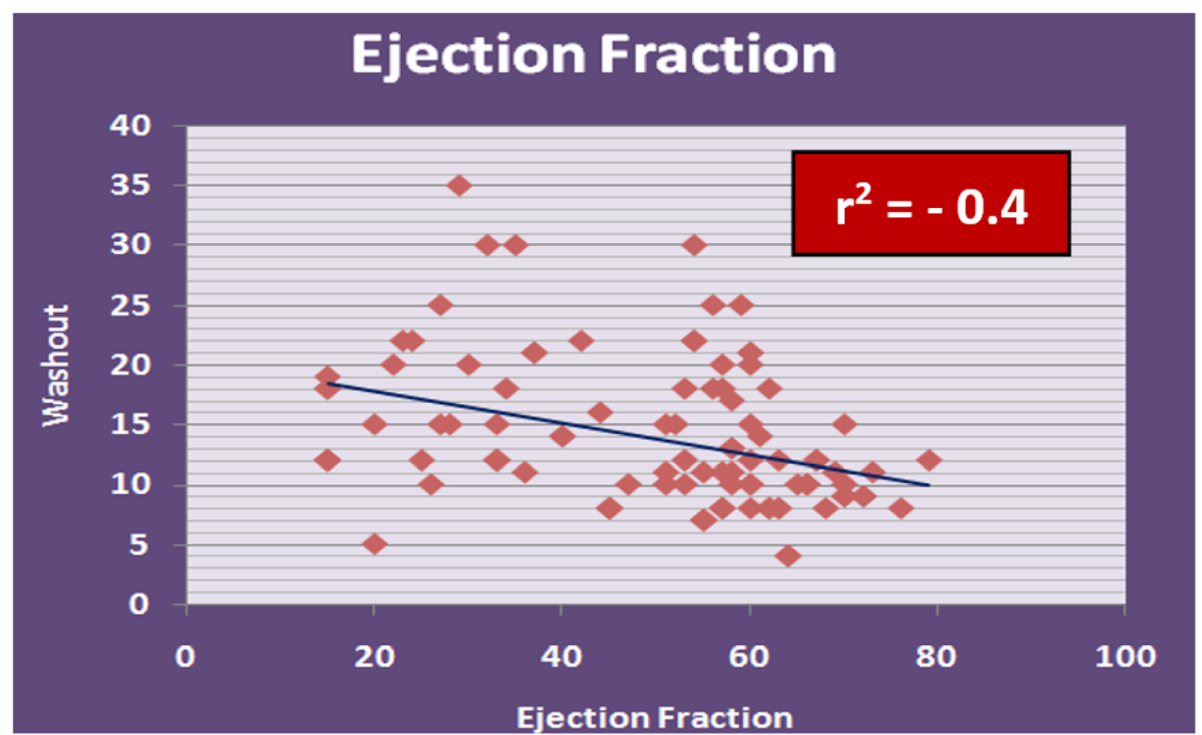

Fig. 4 GWR correlation with EF

In addition, global washout was positively correlated with SDS with correlation coefficient of 0.76 .

Shiroodi et al. studied the ability of GWR to diagnose and evaluate heart failure severity and other left ventricular functional parameters. The study included 17 patients in different stages of dilated cardiomyopathy and decreased ejection fraction. The study demonstrated that GWR correlated with functional cardiac parameters using MPI in patients with idiopathic dilated cardiomyopathy. GWR correlated positively with EDV index and ESV index while it correlated negatively with EF [13].
These findings were close to our results regarding correlation of washout rate to myocardial perfusion findings. GWR correlated positively with ESV and EDV while it was negatively correlated with ejection fraction with correlation coefficient -0.4 .

Omar et al. estimated washout rate in 50 patients referred for MPI. They studied the correlation between the percentages of reversibility and the percentage of washout rate for each vascular territory with significant linear correlation with correlation coefficient for LAD, $\mathrm{LCx}$, and RCA vascular territories was $0.69,0.66$, and 0.77 respectively [14].

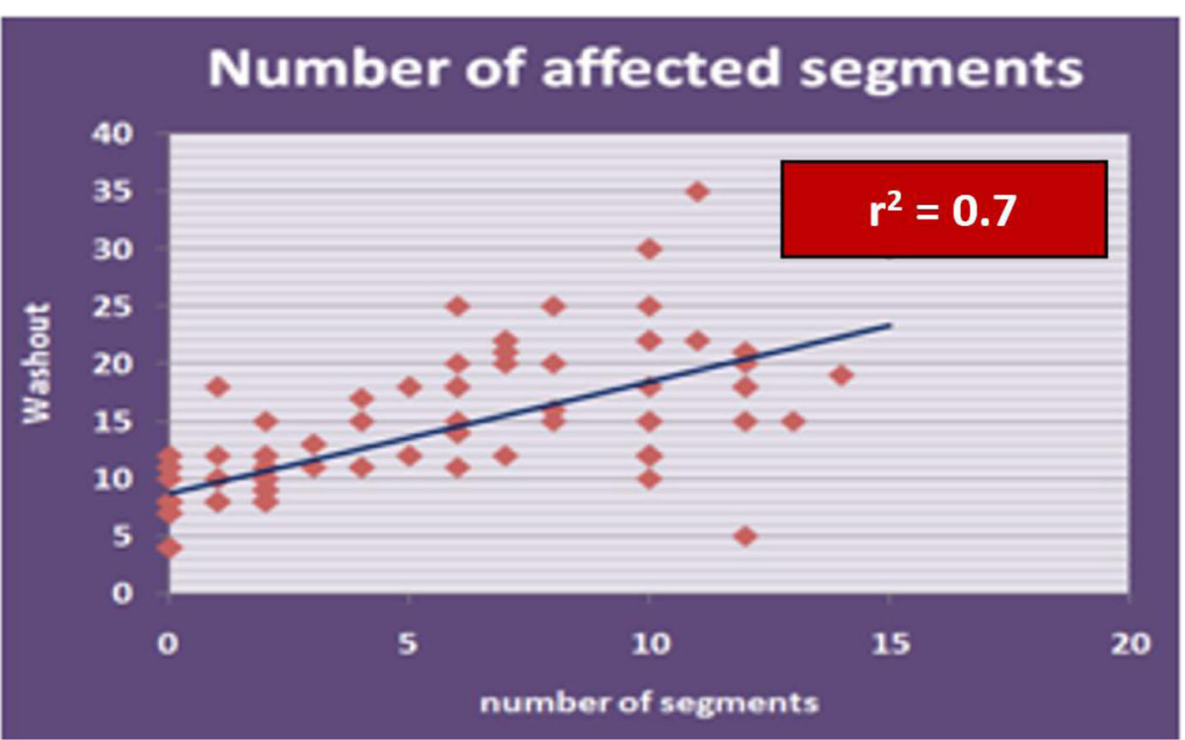

Fig. 5 GWR correlation with affected segment number 


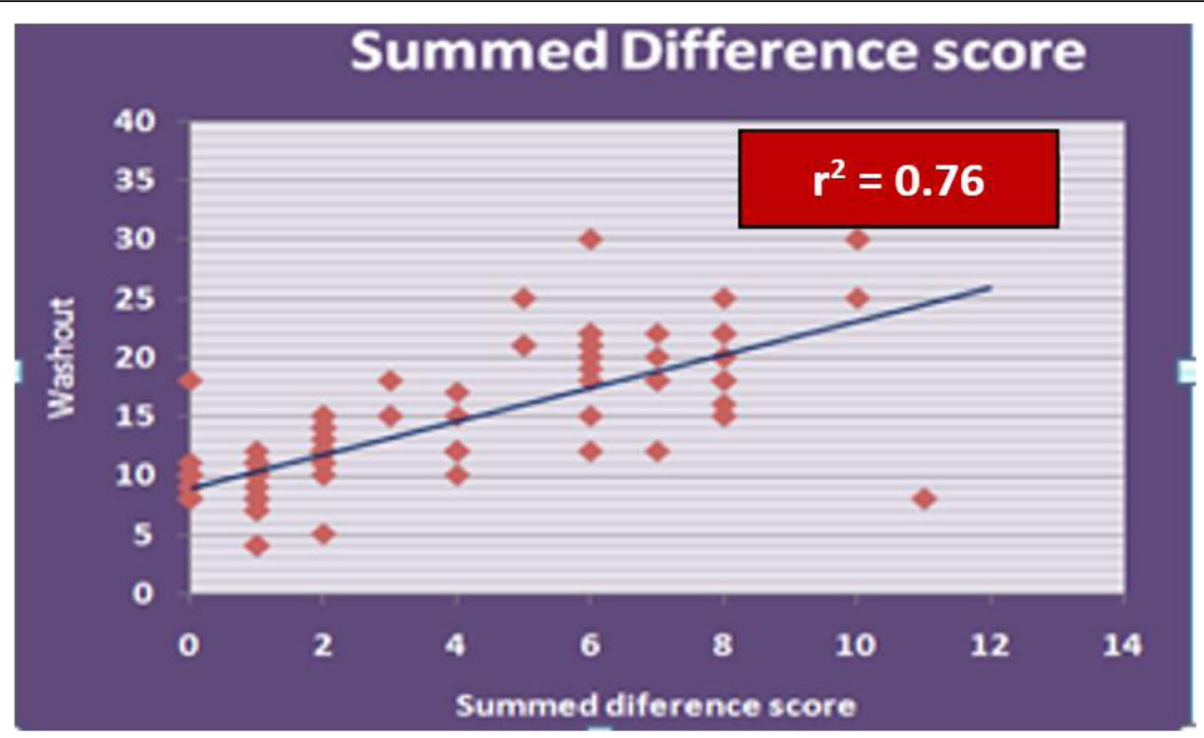

Fig. 6 GWR correlation with SDS

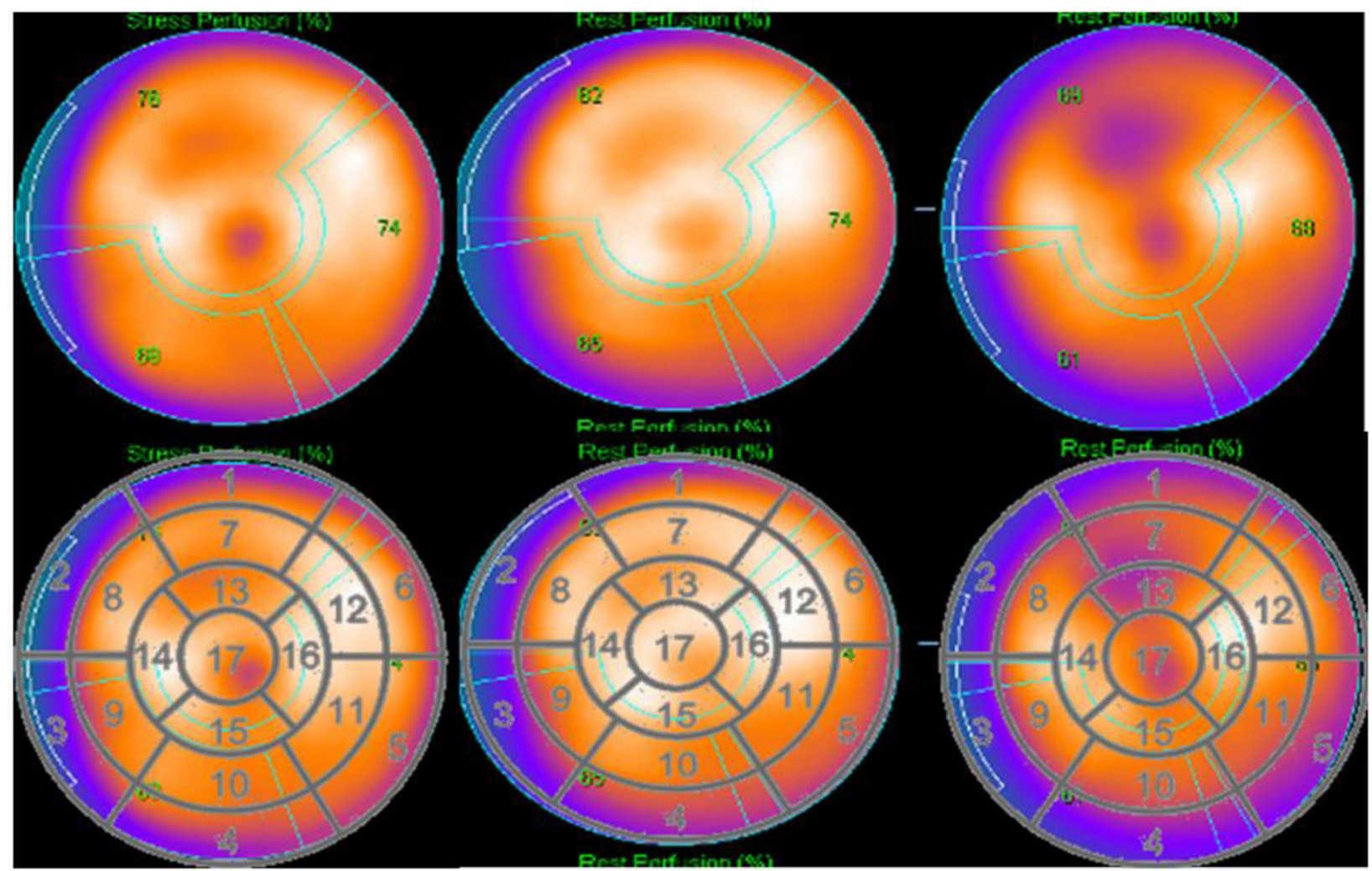

Fig. 7 Polar map in stress, early rest, and delayed rest of MPS of 65-year-old female patient presented with dyspnea, diabetic but not hypertensive classified clinically to be low risk. Her perfusion scan demonstrated mild small ischemia in the apex in the stress study with complete recovery in the rest phase. SSS $=3$, SRS $=1$, and SDS 2. EF was $62 \%$. Her GWR was $8 \%$ 


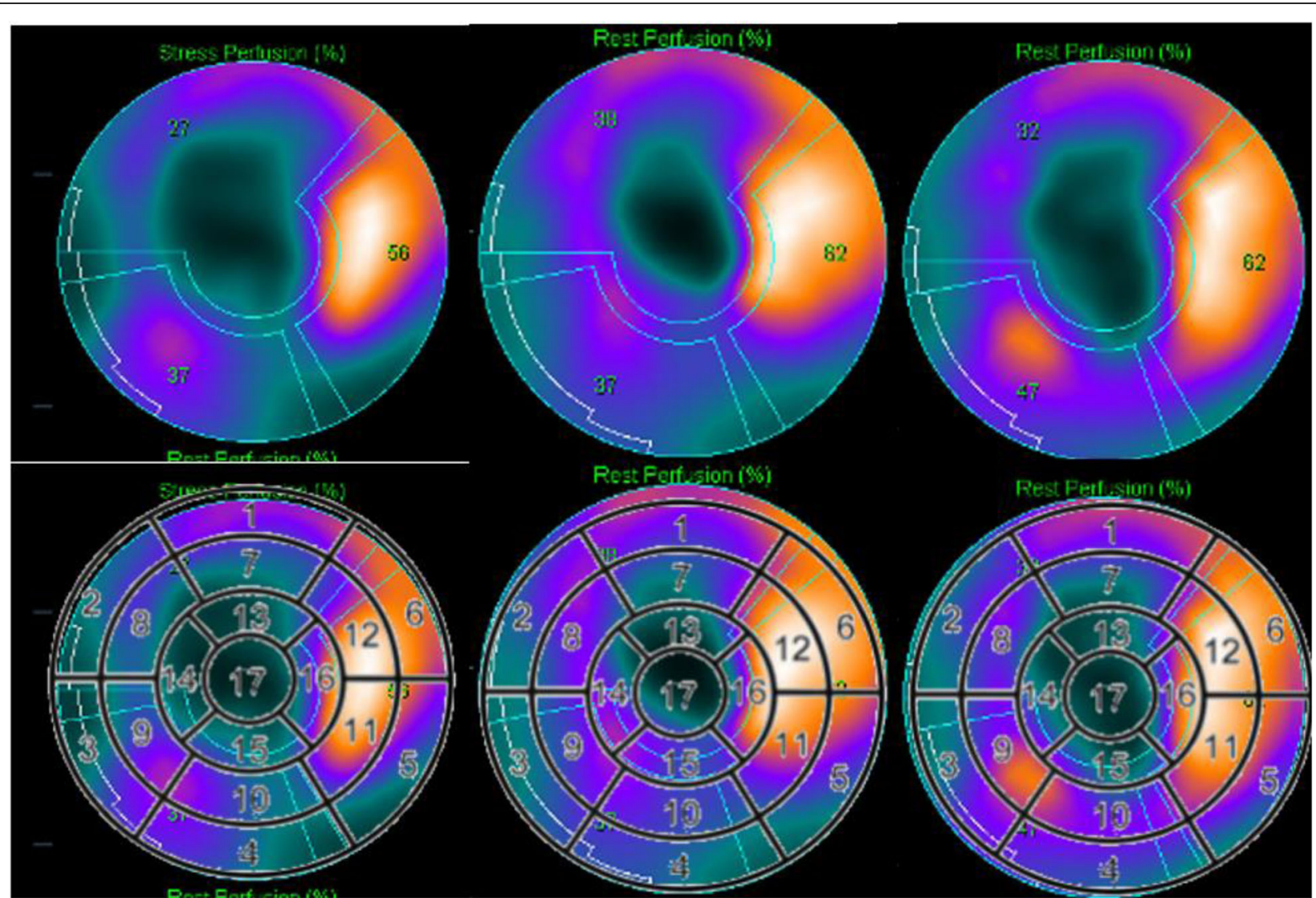

Fig. 8 Polar map in stress, early rest, and delayed rest of MPS of 62-year-old male patient presented with severe chest pain radiating to the back, diabetic, hypertensive, and smoker classified clinically to be high risk. His perfusion scan demonstrated large severe hypoperfusion in the stress study involving the apex as well as the apical and mid segments of the anterior wall. The latter demonstrated partial recovery in the rest phase. SSS $=18$, SRS $=12$, and SDS 6 . EF was $32 \%$. Her GWR was $23 \%$

Also, Du B et al. in 2014 studied seven patients to reveal the concealed balanced ischemia diagnosed by angiography. They reported that significantly higher global washout rate was observed in the three vessels CAD group $(21.1 \pm 4.6 \%)$ than the control group $(9.5 \pm 4.9 \%)$ [15].

\section{Limitations}

Unfortunately, in this study, we had a relatively small number of subjects to evaluate overall pattern of MIBI washout rate in different CAD patients. Also, we had no follow-up for the patients to study myocardial washout rate as an independent poor prognostic factor.

\section{Conclusion}

GWR can be used as an additional parameter for risk stratification of CAD patients. Also, GWR is correlated with the poor prognostic finding in myocardial perfusion scan being most evident with SDS.

\section{Abbreviations}

CAD: Coronary artery disease; MPI: Myocardial perfusion imaging;

GWR: Global myocardial washout rate; LVEF: Left ventricular ejection fraction; SDS: Summed difference score; SPECT: Single-photon computed tomography

\section{Acknowledgements}

Not applicable.

\section{Authors' contributions}

M.O.M.O and H.M.M. put the idea of the study, editors of the manuscript, and participated in the study design. M.O.M.O and S.A.A.E. participated in the study design and performed the statistical analysis. M.O.M.O and M.M.A. contributed to the patients' collection and clinical assessment. The authors read and approved the final manuscript.

\section{Funding}

Not applicable (no funding received for this study).

\section{Availability of data and materials}

All the datasets used and analyzed in this study are available with the corresponding author on reasonable request.

\section{Ethics approval and consent to participate}

The study was approved by the research committee of Faculty of Medicine, Kasr Alainy Hospital, Cairo University, 2017. No reference number provided as the committee just say yes or no according to the system in our faculty of medicine at 2017 (date of starting of this research). All patients included in this study gave written informed consent to participate in this research. If the patients were disoriented about the setting at the time of the study, written informed consent for their participation was given by their legal guardian. 


\section{Consent for publication}

All patients included in this research were fully conscious and gave written informed consent to publish the data contained within this study.

\section{Competing interests}

The authors declare that they have no competing interests.

\section{Author details}

${ }^{1} N$ uclear Medicine, El-Kasr Al-Ainy Cairo University, 20 El-Enshirah El-Kabeer street, El-Mohandeseen, Giza, Egypt. ${ }^{2}$ Cardiology, El-Kasr Al-Ainy Cairo University, 20 El-Enshirah El-Kabeer street, El-Mohandeseen, Giza, Egypt.

Received: 5 August 2020 Accepted: 6 December 2020

Published online: 08 March 2021

\section{References}

1. Kannel W (1994) Incidence, prevalence, and mortality of cardiovascular diseases. In: Hurst's the heart

2. Kannel WB (1987) Prevalence and clinical aspects of unrecognized myocardial infarction and sudden unexpected death. Circulation. 75:|14-115

3. Cremer P, Hachamovitch R, Tamarappoo B (2014) Clinical decision making with myocardial perfusion imaging in patients with known or suspected coronary artery disease. Semin Nucl Med 44(4):320-329

4. D'Agostino RB, Vasan RS, Pencina MJ, Wolf PA, Cobain M, Massaro JM et al (2008) General cardiovascular risk profile for use in primary care. Circulation. 117(6):743-753

5. Fihn SD, Gardin JM, Abrams J, Berra K, Blankenship JC, Douglas PS et al (2012) 2012 ACCF/AHA/ACP/AATS/PCNA/SCAI/STS guideline for the diagnosis and management of patients with stable ischemic heart disease. J Am Coll Cardiol 60(24):e44-e164

6. Czaja M, Wygoda Z, Duszańska A et al (2017) Interpreting myocardial perfusion scintigraphy using single-photon emission computed tomography. Part 1. Kardiochirurgia i Torakochirurgia Polska= Polish J Cardio-Thoracic Surg 14(3):192

7. Kwok JM, Miller TD, Hodge DO, Gibbons RJ (2002) Prognostic value of the Duke treadmill score in the elderly. J Am Coll Cardiol 39(9):1475-1481

8. Knuuti J, Wijns W, Saraste A et al (2020) 2019 ESC guidelines for the diagnosis and management of chronic coronary syndromes: the task force for the diagnosis and management of chronic coronary syndromes of the European Society of Cardiology (ESC). Eur Heart J 41(3):407-477

9. Montalescot G, Sechtem U, Achenbach S, Andreotti F, Arden C, Budaj A et al (2013) 2013 ESC guidelines on the management of stable coronary artery disease: the task force on the management of stable coronary artery disease of the European Society of Cardiology. Eur Heart J 34(38):2949-3003

10. Richter W-S, Cordes M, Calder D, Eichstaedt H, Felix R (1995) Washout and redistribution between immediate and two-hour myocardial images using technetium-99m sestamibi. Eur J Nucl Med 22(1):49-55

11. Khandaker MH, Miller TD, Chareonthaitawee P, Askew JW, Hodge DO, Gibbons RJ (2009) Stress single photon emission computed tomography for detection of coronary artery disease and risk stratification of asymptomatic patients at moderate risk. J Nucl Cardiol 16(4):516-523

12. America YG, Bax JJ, Boersma E, Stokkel M, van der Wall EE (2009) The additive prognostic value of perfusion and functional data assessed by quantitative gated SPECT in women. J Nucl Cardiol 16(1):10-19

13. Shiroodi MK, Shafiei B, Baharfard N, Gheidari ME, Nazari B, Pirayesh E et al (2012) Tc99m MIBI washout as a complementary factor in the evaluation of idiopathic dilated cardiomyopathy (IDCM) using myocardial perfusion imaging. Int J Cardiovasc Imaging 28(1):211-217

14. Omar M, Moustafa H (2014) Myocardial 99TC-MIBI washout. Egyptian J Nucl Med 10(2):1

15. Du B, Li N, Li X et al (2014) Myocardial washout rate of resting 99m Tc-Sestamibi (MIBI) uptake to differentiate between normal perfusion and severe three-vessel coronary artery disease documented with invasive coronary angiography. Ann Nucl Med 28(3):285-292

\section{Publisher's Note}

Springer Nature remains neutral with regard to jurisdictional claims in published maps and institutional affiliations.

\section{Submit your manuscript to a SpringerOpen ${ }^{\circ}$ journal and benefit from:}

- Convenient online submission

- Rigorous peer review

- Open access: articles freely available online

- High visibility within the field

- Retaining the copyright to your article

Submit your next manuscript at $\boldsymbol{\nabla}$ springeropen.com 